TRANSACTIONS OF THE

AMERICAN MATHEMATICAL SOCIETY

Volume 326, Number 2, August 1991

\title{
MONOGENIC DIFFERENTIAL CALCULUS
}

\author{
F. SOMMEN
}

\begin{abstract}
In this paper we study differential forms satisfying a Dirac type equation and taking values in a Clifford algebra. For them we establish a Cauchy representation formula and we compute winding numbers for pairs of nonintersecting cycles in $\mathbb{R}^{m}$ as residues of special differential forms. Next we prove that the cohomology spaces for the complex of monogenic differential forms split as direct sums of de Rham cohomology spaces. We also study duals of spaces of monogenic differential forms, leading to a general residue theory in Euclidean space. Our theory includes the one established in our paper [11] and is strongly related to certain differential forms introduced by Habetha in [4].
\end{abstract}

\section{INTRODUCTION}

In [2], a function theory was built up for Clifford algebra valued functions in $\mathbb{R}^{m+1}$ satisfying $\left(\partial_{x_{0}}+\partial_{\mathbf{x}}\right) f=0, \partial_{\mathbf{x}}$ the Dirac operator. When such a so-called monogenic function $f$ is independent of the $x_{0}$-variable, it may be regarded as a solution of $\partial_{\mathbf{x}} f=0$ in some domain of $\mathbb{R}^{m}$. In this paper we only consider those. One of the simplest but most frequently used properties of such functions is Cauchy's theorem, which is essentially based on the fact that the differential form $d \sigma_{\mathbf{x}} f(x), d \sigma_{\mathbf{x}}=\sum_{j=1}^{m}(-1)^{j+1} e_{j} d \hat{x}_{j}$, is closed if and only if $f$ is left monogenic, i.e., $\partial_{\mathbf{x}} f=0$. Hence Cauchy's theorem involves certain integrals of monogenic functions over cycles of dimension $m-1$, but within the theory developed in [2], no version of this theorem seems to exist for cycles of any dimension $k$. Of course there is no such incompleteness in the case of holomorphic functions in the plane (i.e., $m=2$ ) because there the only nontrivial cycles are 1-cycles. As to the general case, it is classically known that to each Clifford function $f$ one can associate in a 1-1 way a differential form $f_{0}+\cdots+f_{m}, f_{k}$ being a $k$-form (see also [5]). The Dirac operator may then be written as $\partial_{\mathbf{x}}=d-d^{*}, d$ being the exterior derivative and $d^{*}$ its "Hodge dual" (see [7]). Hence $f_{k}$ is left monogenic if and only if $d f_{k}=d^{*} f_{k}=0$, i.e., $f_{k}$ is a harmonic differential form in Hodge's sense. Of course there is the whole theory of harmonic integrals developed for such differential forms in

Received by the editors July $18,1989$.

1980 Mathematics Subject Classification (1985 Revision). Primary 30G35, 46F15, 55M25, $58 \mathrm{~A} 12,58 \mathrm{~A} 14$.

The author is a research associate supported by the National Fund for Scientific Research, Belgium. 
[7], involving cycles of any dimension; but this is not equivalent to Cauchy's theorem for the differential forms $d \sigma_{\mathbf{x}}$ in the case $k=m-1$. Mittag-Leffler's theorem for example is valid for monogenic functions but not for harmonic differential forms. Of course one may split $d \sigma_{\mathbf{x}} f(x)$ into harmonic forms, but the pieces are linked together in a special way.

To give an answer to this problem, in [11] we introduced a concept of monogenic differential forms starting from the hypercomplex differential forms $d z_{j}=d x_{j}-e_{j} d x_{0}$, which generalize $d z=d x+i d y$ in the plana Using these differential forms, one can compute winding numbers and represent the de Rham cohomology spaces (see also [1, 6-9]). Unfortunately this theory is only coordinate independent for $(m-1)$-forms, so that it cannot work on manifolds. Other splittings of the de Rham complex were studied in [12, 13], but except for the above theory [11], up to now there are no formulae for winding numbers available. In the case of $(m-1)$-forms, winding number formulae were also obtained by Hestenes in [5] and Habetha in [4]. Although Habetha only studied $(m-1)$-cycles, his paper contains special differential forms $\omega_{A}$ which fit completely in the theory developed here.

Our present theory is designed as follows. We first interpret the Dirac operator as a Clifford algebra valued vector field $\partial_{\mathbf{x}}$ and so we can consider the contraction $\left.\partial_{\mathbf{x}}\right\rfloor F$ of $\partial_{\mathbf{x}}$ with a Clifford algebra valued differential form $F$. The Dirac operator $\partial_{\mathbf{x}} F$ is defined as a Lie-derivative by (see also [14])

$$
\left.\left.\partial_{\mathbf{x}} F=d \wedge\left(\partial_{\mathbf{x}}\right\rfloor F\right)+\partial_{\mathbf{x}}\right\rfloor(d \wedge F) .
$$

Next we generalize the differential forms $f(\mathbf{x}) d \sigma_{\mathbf{x}} g(x)$ by considering the so-called monogenic coupling

$$
\left.\left.F \diamond G=(F\rfloor \partial_{\mathbf{x}}\right) \wedge G-F \wedge\left(\partial_{\mathbf{x}}\right\rfloor G\right)
$$

and we prove the basic identity

$$
d(F \diamond G)=(d F) \diamond G+(-1)^{k-1} F \diamond(d G)+(-1)^{k-1}\left(F \partial_{\mathbf{x}} \wedge G+F \wedge \partial_{\mathbf{x}} G\right) .
$$

This identity plays an essential role for the Cauchy-Pompeiu formula which we formulate in $\S 2$. In $\S 3$ we construct for a given $(m-k)$-chain $\Sigma$ in $\mathbb{R}^{m}$ its indicatrix $I_{l}(\Sigma)(\mathbf{x})$, which is a left monogenic differential form satisfying

$$
d \wedge I_{l}(\Sigma)(\mathbf{x})=(-1)^{k+1} I_{l}(\partial \Sigma)(\mathbf{x}), \quad \mathbf{x} \in \mathbb{R}^{m} \backslash \Sigma .
$$

Hence, when $\Sigma$ is a cycle, then $I_{l}(\Sigma)$ is closed. Furthermore when $\Sigma^{\prime}$ is a $(k-1)$-cycle which does not intersect $\Sigma$, then we prove that, up to a signature,

$$
\left.I\left(\Sigma^{\prime}, \Sigma\right)=\int_{\Sigma^{\prime}} \partial_{\mathbf{x}}\right\rfloor I_{l}(\Sigma)(\mathbf{x})
$$

is the winding number of $\Sigma$ and $\Sigma^{\prime}$ which is defined as the intersection number $\tau\left(\Sigma^{\prime \prime}, \Sigma\right), \Sigma^{\prime}=\partial \Sigma^{\prime \prime}$ (see also $[6,8]$ ).

In $\S 4$ we prove that the cohomology spaces $H_{l}^{k}(\Omega)$, arising from the complex of monogenic differential forms in $\Omega \subseteq \mathbb{R}^{m}$ open, admits the splitting into 
de Rham cohomology spaces

$$
H_{l}^{k}(\Omega) \cong H_{\mathrm{deRham}}^{k}(\Omega) \oplus H_{\mathrm{deRham}}^{k-1}(\Omega)
$$

Next, in $\S 5$ we develop a duality theory for various spaces of monogenic differential forms, thus generalizing the paper [3]. We also define the notion of "grand residue" of a monogenic form, which includes various type of compactly supported distributional currents (see also [9]).

Finally, we show that our previous theory [11] is included in the present one. We also show that the identity for monogenic forms $\left.d\left(\partial_{\mathbf{x}}\right\rfloor F\right)=-\partial_{\mathbf{x}}(d F)$ is already hidden in a lemma proved by Habetha in [4].

Preliminaries. $\mathbb{C}_{m}$ is the complex Clifford algebra constructed with an orthonormal basis $\left\{e_{1}, \ldots, e_{m}\right\}$ of $\mathbb{R}^{m}$, i.e., $a \in \mathbb{C}_{m}$ is of the form $a=\sum_{A \subseteq M} a_{A} e_{A}$, $M=\{1, \ldots, m\}, a_{A} \in \mathbb{C}$, where $e_{\varnothing}=1, e\{j\}=e_{j}$, and $e_{A}=e_{\alpha_{1}} \cdots e_{\alpha_{k}}$ for $A=\left\{\alpha_{1}, \ldots, \alpha_{k}\right\}$ with $\alpha_{1}<\cdots<\alpha_{k}$. The product is determined by $e_{i} e_{j}+e_{j} e_{i}=-2 \delta_{i j}$, i.e., when $\mathbf{x}, \mathbf{y} \in \mathbb{R}^{m} \subseteq \mathbb{C}_{m}, \mathbf{x y}=-\mathbf{x} \cdot \mathbf{y}+\mathbf{x} \wedge \mathbf{y}$ with $\mathbf{x} \cdot \mathbf{y}=\sum_{1}^{m} x_{j} y_{j}, \mathbf{x} \wedge \mathbf{y}=\sum_{i<j} e_{i j}\left(x_{i} y_{i}-x_{i} y_{i}\right)$. Let $\partial_{\mathbf{x}}=\sum_{j=1}^{m} e_{j} \partial_{x_{j}}$ be the Dirac operator; then solutions of $\partial_{\mathbf{x}} f=0$ (resp. $f \partial_{\mathbf{x}}=0$ ) are called left (resp. right) monogenic functions. Throughout this paper we consider $\mathbb{C}_{m^{-}}$ valued differential forms $F(\mathbf{x})=\sum_{A} d x_{A} F_{A}(\mathbf{x}), d x_{A}=d x_{\alpha_{1}} \wedge \cdots \wedge d x_{\alpha_{k}}, F_{A}$ being $\mathbb{C}_{m}$-valued functions. For a "vector field" $t=\sum t_{j} \partial_{x_{j}}$, the contraction $t\rfloor F$ is given in the usual way by $\left.t\rfloor F=\sum_{j, A} t_{j} \partial_{x_{j}}\right\rfloor d x_{A} F_{A}$ with $\left.\partial_{x_{j}}\right\rfloor d x_{A}=$ $\sum_{l=1}^{k}(-1)^{l+1} \delta_{j \alpha_{l}} d x_{A \backslash\left\{\alpha_{l}\right\}}$. We also denote $\left.\left.F\right\rfloor t=(-1)^{k-1} t\right\rfloor F$. For further details on differential forms, see, e.g., [14]. For Clifford analysis, see [2, 4, 5, 10-13].

\section{MoNOGENIC DIFFERENTIAL FORMS}

Consider the Dirac operator $\partial_{\mathbf{x}}=\sum_{j=1}^{m} e_{j} \partial_{x_{j}}$. Then a $\mathbb{C}_{m}$-valued function $f(\mathbf{x}), \mathbf{x} \in \Omega \subseteq \mathbb{R}^{m}$ open, is called left (right) monogenic if $\partial_{\mathbf{x}} f=0$ (resp. $\left.f \partial_{\mathbf{x}}=0\right)$ in $\Omega$.

Next, consider a $\mathbb{C}_{m}$-valued differential $k$-form $F$. Then, as $\partial_{\mathbf{x}}$ may be interpreted as a $\mathbb{C}_{m}$-valued vector field, it makes sense to consider the contraction $\left.\partial_{\mathbf{x}}\right\rfloor F$, which is a differential $(k-1)$-form. Of course we can also consider the contraction $F\rfloor \partial_{\mathbf{x}}$ from the right.

Examples. (1) Let $F=d x_{1} \wedge \cdots \wedge d x_{m} f(x)$; then $\left.\partial_{\mathbf{x}}\right\rfloor F=d \sigma_{\mathbf{x}} f(\mathbf{x})$, where

$$
d \sigma_{\mathbf{x}}=\sum_{j=1}^{m}(-1)^{j+1} e_{j} d \hat{x}_{j}, \quad d \hat{x}_{j}=d x_{1} \wedge \cdots \wedge d x_{j-1} \wedge d x_{j+1} \wedge \cdots \wedge d x_{m}
$$

is the basic differential form commonly used in quaternion and Clifford analysis (see, e.g., $[2,5])$.

(2) Let $F=\sum_{j=1}^{m} d x_{j} f_{j}(\mathbf{x})$; then $\left.\partial_{\mathbf{x}}\right\rfloor F=\sum_{j=1}^{m} e_{j} f_{j}$. 
(3) Let $F=\sum_{j<k} d x_{j} \wedge d x_{k} f_{j k}(\mathbf{x})$; then

$$
\left.\partial_{\mathbf{x}}\right\rfloor F=\sum_{j<k}\left(e_{j} d x_{k}-e_{k} d x_{j}\right) f_{j k} .
$$

(4) In general, we have that for $\left.F=\sum_{|A|=k} d x_{A} f_{A}, \partial_{\mathbf{x}}\right\rfloor F=\sum_{A} d \sigma_{A} f_{A}$, where for $A=\left\{\alpha_{1}, \ldots, \alpha_{k}\right\}$ with $\alpha_{1}<\cdots<\alpha_{k}, d \sigma_{A}=\sum_{j=1}^{k}(-1)^{j+1} e_{\alpha_{j}} d \hat{x}_{\alpha_{j}}$ $\left.=\sum_{j=1}^{m} e_{j}\left(\partial_{x_{j}}\right\rfloor d x_{A}\right)$ is the basic Clifford differential form in $\mathbb{R}^{k}$.

The differential forms $d \sigma_{x} f(\mathbf{x})$ are most commonly used in Clifford analysis and many function theoretic properties follow from the simple fact that such a differential form is closed if and only if $f$ is left monogenic, i.e., $\partial_{\mathbf{x}} f=0$. Indeed we have that

$$
d_{\mathbf{x}} \wedge d \sigma_{\mathbf{x}} f(\mathbf{x})=d x_{M} \partial_{\mathbf{x}} f(x),
$$

where $M=\{1, \ldots, m\}, d x_{M}=d x_{1} \wedge \cdots \wedge d x_{m}, d_{\mathbf{x}}=\sum_{j=1}^{m} d x_{j} \partial_{x_{j}}$. Hence, if we are to generalize Clifford analysis, to incorporate differential forms of any type, we have to know $\left.d_{\mathbf{x}} \wedge\left(\partial_{\mathbf{x}}\right\rfloor F\right)$. Now we have that

$$
\begin{aligned}
\left.\partial_{\mathbf{x}}\right\rfloor\left(d_{\mathbf{x}} \wedge F\right) & \left.=\sum_{j=1}^{m} e_{j}\left(\partial_{x_{j}}\right\rfloor\left(d_{\mathbf{x}} \wedge F\right)\right) \\
& \left.\left.=\sum_{j=1}^{m} e_{j}\left(\partial_{x_{j}}\right\rfloor d_{\mathbf{x}}\right) F-\sum_{j=1}^{m} e_{j}\left(d_{\mathbf{x}} \wedge\left(\partial_{x_{j}}\right\rfloor F\right)\right) \\
& \left.=\partial_{\mathbf{x}} F-d_{\mathbf{x}} \wedge\left(\partial_{\mathbf{x}}\right\rfloor F\right),
\end{aligned}
$$

where $\partial_{\mathbf{x}} F=\sum_{A} d x_{A} \partial_{\mathbf{x}} F_{A}$ is in fact the Lie-derivative of $F$ with respect to the vector field $\partial_{\mathbf{x}}$. Hence $\partial_{\mathrm{x}} F$ has an invariant meaning. Similarly we have that $\left.\left.\left(F \wedge d_{\mathbf{x}}\right)\right\rfloor \partial_{\mathbf{x}}=F \partial_{\mathbf{x}}-(F\rfloor \partial_{\mathbf{x}}\right) \wedge d_{\mathbf{x}}$, where $\left.d x_{j}\right\rfloor \partial_{x_{k}}=\delta_{j k}$.

Notice that $\partial_{\mathbf{x}} F=0$ implies that the coefficients of $F$ are left monogenic. It does not imply that $\left.d_{\mathbf{x}} \wedge\left(\partial_{\mathbf{x}}\right\rfloor F\right)=0$ or vice versa. Only if $F$ is closed, the closedness of $\left.\partial_{\mathbf{x}}\right\rfloor F$ is equivalent to the left monogenicity of the coefficients of $F$.

Definition 1. A $\mathbb{C}_{m}$-valued $k$-form $F$ is called left (right) monogenic if $\partial_{\mathbf{x}} F=$ 0 (resp. $F \partial_{\mathbf{x}}=0$ ).

The relations

$$
\begin{aligned}
& \left.\left.\partial_{\mathbf{x}} F=d_{\mathbf{x}} \wedge\left(\partial_{\mathbf{x}}\right\rfloor F\right)+\partial_{\mathbf{x}}\right\rfloor\left(d_{\mathbf{x}} \wedge F\right), \\
& \left.\left.F \partial_{\mathbf{x}}=(F\rfloor \partial_{\mathbf{x}}\right) \wedge d_{\mathbf{x}}+\left(F \wedge d_{\mathbf{x}}\right)\right\rfloor \partial_{\mathbf{x}}
\end{aligned}
$$

are called the first basic identities. In the sequel we will also use the notation $d_{\mathbf{x}} F$ or $d F$ instead of $d_{\mathbf{x}} \wedge F$.

Next, practically all $(m-1)$-dimensional contour integrals involve differential forms of the form $f(\mathbf{x}) d \sigma_{\mathbf{x}} g(\mathbf{x})$, with $d \sigma_{\mathbf{x}}=\sum_{j=1}^{m}(-1)^{j+1} e_{j} d \hat{x}_{j}$, which satisfy the fundamental relation

$$
d_{\mathbf{x}}\left(f(\mathbf{x}) d \sigma_{\mathbf{x}} g(\mathbf{x})\right)=\left(\left(f(\mathbf{x}) \partial_{\mathbf{x}}\right) g(\mathbf{x})+f(\mathbf{x})\left(\partial_{\mathbf{x}} g(\mathbf{x})\right)\right) d x_{M} .
$$


This relation leads to, e.g., Cauchy-Pompeiu type integral formulae, residue calculus, and boundary value theory. In order to generalize such forms, let us first see how we can make them.

First take $F=f, G=d x_{M} g$. Then clearly $\left.f d \sigma_{x} g=F \wedge\left(\partial_{\mathbf{x}}\right\rfloor G\right)$. This formula clearly lacks symmetry and it would be a bad idea to use the expression $\left.F \wedge\left(\partial_{\mathbf{x}}\right\rfloor G\right)$ as a generalization of the above differential forms. Take however $F=f d x_{A}$ and $G=d x_{M \backslash A} g, A=\{1, \ldots, k\}$. Then

$$
\begin{aligned}
f d \sigma_{x} g & \left.=f \partial_{\mathbf{x}}\right\rfloor\left(d x_{A} \wedge d x_{M \backslash A}\right) g \\
& \left.\left.=f\left(\partial_{\mathbf{x}}\right\rfloor d x_{A}\right) \wedge G+(-1)^{k} F \wedge\left(\partial_{\mathbf{x}}\right\rfloor G\right) \\
& \left.\left.=(-1)^{k-1}(F\rfloor \partial_{\mathbf{x}} \wedge G-F \wedge \partial_{\mathbf{x}}\right\rfloor G\right) .
\end{aligned}
$$

This gives us a good way of generalizing $f d \sigma_{x} g$.

Definition 2. Let $F$ be a $k$-form and $G$ an $l$-form. Then the monogenic coupling of $F$ and $G$ is defined by $\left.F \diamond G=F\rfloor \partial_{\mathbf{x}} \wedge G-F \wedge \partial_{\mathbf{x}}\right\rfloor G$.

We immediately have the following

Theorem 1 (Second basic identity). Let $F$ be a k-form and $G$ an l-form with $k+l \leq m$. Then we have $d_{\mathbf{x}}(F \diamond G)=\left(d_{\mathbf{x}} F\right) \diamond G+(-1)^{k-1} F \diamond\left(d_{\mathbf{x}} G\right)+$ $(-1)^{k-1}\left[F \partial_{\mathbf{x}} \wedge G+F \wedge \partial_{\mathbf{x}} G\right]$. When $k+l=m$, this identity can be simplified to the so-called "duality principle"

$$
d_{\mathbf{x}}(F \diamond G)=(-1)^{k-1}\left[F \partial_{\mathbf{x}} \wedge G+F \wedge \partial_{\mathbf{x}} G\right]
$$

Proof. The second basic identity for $k+l<m$ is easy to verify, using the first basic identities and the definition of $F \diamond G$. As to the duality principle, let, e.g., $A=\{1, \ldots, k\}$ and $B=\{m-l+1, \ldots, m\}$ such that $k+l>m$ and put $C=A \cap B=\{m-l+1, \ldots, k\}$. When $j \in A \backslash C$, then $\left.\partial_{x_{j}}\right\rfloor d x_{B}=0$ and

$$
\left.\left.d x_{A}\right\rfloor \partial_{x_{j}}=(-1)^{m-k-l}\left(d x_{A \backslash C}\right\rfloor \partial_{x_{j}}\right) \wedge d x_{C},
$$

so that clearly $\left.\left.\left(d x_{A}\right\rfloor \partial_{x_{j}}\right) \wedge d x_{B}-d x_{A} \wedge\left(\partial_{x_{j}}\right\rfloor d x_{B}\right)=0$. A similar identity holds for $j \in B \backslash C$.

When $j \in C$, then $\left.d x_{A \backslash C} \wedge\left(d x_{C}\right\rfloor \partial_{x_{j}}\right) \wedge d x_{B}$ and $\left.d x_{A} \wedge\left(\partial_{x_{j}}\right\rfloor d x_{C} \wedge d x_{B \backslash C}\right)$ clearly vanish when $|C|>1$. When $C=\{k\}$, then $\left.d x_{A}\right\rfloor \partial_{x_{k}}=d x_{A \backslash C}$ and $\left.\partial_{x_{k}}\right\rfloor d x_{B}=d x_{B \backslash C}$ so that

$$
\left.\left.d x_{A}\right\rfloor \partial_{x_{k}} \wedge d x_{B}-d x_{A} \wedge \partial_{x_{k}}\right\rfloor d x_{B}=d x_{M}-d x_{M}=0 .
$$

This implies that when $k+l>m$, then always $F \diamond G=0$, so that for $k+l=m$, the second basic identity leads to the duality principle.

Notice that when $F$ is right monogenic and $G$ left monogenic, then

$$
d_{\mathbf{x}}(F \diamond G)=\left(d_{\mathbf{x}} F\right) \diamond G+(-1)^{k-1} F \diamond\left(d_{\mathbf{x}} G\right)
$$

while for $k+l=m, F \diamond G$ is closed. 


\section{The Cauchy-Pompeiu formula}

The duality principle will now be used to establish a Cauchy-Pompeiu type formula for differential forms. First, recall that the Cauchy kernel is given by

$$
E(\mathbf{x}-\mathbf{y})=\frac{1}{\omega_{m}} \frac{\mathbf{x}-\mathbf{y}}{|\mathbf{x}-\mathbf{y}|^{m}}, \quad \mathbf{x} \neq \mathbf{y} .
$$

$E(\mathbf{x}-\mathbf{y})$ is left and right monogenic in $\mathbf{x}$ and $\mathbf{y}$ for $\mathbf{x} \neq \mathbf{y}$ and satisfies the equation

$$
\partial_{\mathbf{y}} E(\mathbf{x}-\mathbf{y})=E(\mathbf{x}-\mathbf{y}) \partial_{\mathbf{y}}=\delta(\mathbf{x}-\mathbf{y}),
$$

i.e., it is the fundamental solution.

To make monogenic differential forms out of that, we introduce the "volume elements"

$$
d V_{k}(\mathbf{x}, \mathbf{y})=\sum_{|A|=k} \operatorname{sgn} A d x_{A} d y_{M \backslash A},
$$

where $A=\left\{\alpha_{1}, \ldots, \alpha_{k}\right\}, M \backslash A=\left\{\beta_{1}, \ldots, \beta_{m-k}\right\}$ with $\alpha_{1}<\cdots<\alpha_{k}$ and $\beta_{1}<\cdots<\beta_{m-k}$, and where $\operatorname{sgn} A$ is the signature of the permutation $\left\{\alpha_{1}, \ldots, \alpha_{k}, \beta_{1}, \ldots, \beta_{m-k}\right\}$ of $M$. First we have the following

Lemma 1. For every $k$-form with continuous coefficients,

$$
\int_{\mathbb{R}^{m}} \delta(\mathbf{x}-\mathbf{y}) d V_{k}(\mathbf{x}, \mathbf{y}) \wedge F(\mathbf{y})=(-1)^{k(m-k)} F(\mathbf{x}) .
$$

Proof. Take, e.g., $F=d x_{A}$; then the left-hand side equals

$$
\begin{aligned}
\int_{\mathbb{R}^{m}} \delta(\mathbf{x}-\mathbf{y}) \operatorname{sgn} A d x_{A} d y_{M \backslash A} \wedge d y_{A} \\
\quad=\operatorname{sgn} A \operatorname{sgn} M \backslash A d x_{A} \int_{\mathbb{R}^{m}} \delta(\mathbf{x}-\mathbf{y}) d y_{M}=\operatorname{sgn} A \operatorname{sgn} M \backslash A d x_{A}
\end{aligned}
$$

and $\operatorname{sgn} A=(-1)^{k(m-k)} \operatorname{sgn} M \backslash A$.

Of course we also have that for $(m-k)$-forms

$$
\int_{\mathbb{R}^{m}} \delta(\mathbf{x}-\mathbf{y}) G(\mathbf{x}) \wedge d V_{k}(\mathbf{x}, \mathbf{y})=(-1)^{k(m-k)} G(\mathbf{y}) .
$$

We now come to

Theorem 2 (Cauchy-Pompeiu). Let $F$ be a $k$-form with $C_{1}$-coefficients in an open set $\Omega$ and let $C \subseteq \Omega$ be compact with piecewise smooth boundary. Then

$$
\begin{array}{rl}
\int_{\partial C} & E(\mathbf{x}-\mathbf{y}) d V_{k}(\mathbf{x}, \mathbf{y}) \diamond F(y) \\
& =(-1)^{k-1} \int_{C} E(\mathbf{x}-\mathbf{y}) d V_{k}(\mathbf{x}, \mathbf{y}) \wedge \partial_{\mathbf{y}} F(\mathbf{y})-(-1)^{k(m-k-1)} F(\mathbf{x}) .
\end{array}
$$

The proof follows from Stokes' theorem, Lemma 1, and the fact that

$$
\begin{aligned}
& d_{\mathbf{x}}\left(E(\mathbf{x}-\mathbf{y}) d V_{k}(\mathbf{x}, \mathbf{y}) \diamond F(\mathbf{y})\right) \\
& \quad=(-1)^{k-1}\left(\delta(\mathbf{x}-\mathbf{y}) d V_{k}(\mathbf{x}, \mathbf{y}) \wedge F(\mathbf{y})+E(\mathbf{x}-\mathbf{y}) d V_{k}(\mathbf{x}, \mathbf{y}) \wedge \partial_{\mathbf{x}} F(\mathbf{y})\right) .
\end{aligned}
$$


Similarly, for an $(m-k)$-form $G(\mathbf{x})$,

$$
\begin{aligned}
& d_{\mathbf{x}}\left(G(\mathbf{x}) \diamond d V_{k}(\mathbf{x}, \mathbf{y}) E(\mathbf{y}-\mathbf{x})\right) \\
& \quad=(-1)^{m-k-1}\left(G(\mathbf{x}) \partial_{\mathbf{x}} \wedge d V_{k}(\mathbf{x}, \mathbf{y}) E(\mathbf{y}-\mathbf{x})+\delta(\mathbf{x}-\mathbf{y}) G(\mathbf{x}) \wedge d V_{k}(\mathbf{x}, \mathbf{y})\right)
\end{aligned}
$$

so that also

$$
\begin{array}{rl}
\int_{\partial C} & G(\mathbf{x}) \diamond d V_{k}(\mathbf{x}, \mathbf{y}) E(\mathbf{y}-\mathbf{x}) \\
& =(-1)^{m-k-1} \int_{G} G(\mathbf{x}) \partial_{\mathbf{x}} \wedge d V_{k}(\mathbf{x}, \mathbf{y}) E(\mathbf{y}-\mathbf{x})-(-1)^{(m-k)(k-1)} G(\mathbf{y}) .
\end{array}
$$

Corollary (Cauchy's representation formula). Let $F$ be a left monogenic $k$-form and $G$ a right monogenic $(m-k)$-form in $\Omega$. Then we have that for $\mathbf{x} \in \stackrel{\circ}{C}$ (resp. $\mathbf{y} \in \stackrel{\circ}{\mathrm{C}}$ )

$$
\begin{aligned}
& F(\mathbf{x})=(-1)^{k(m-k-1)} \int_{\partial C} E(\mathbf{y}-\mathbf{x}) d V_{k}(\mathbf{x}, \mathbf{y}) \diamond F(\mathbf{y}), \\
& G(\mathbf{y})=(-1)^{(m-k)(k-1)} \int_{\partial C} G(\mathbf{x}) \diamond d V_{k}(\mathbf{x}, \mathbf{y}) E(\mathbf{x}-\mathbf{y}) .
\end{aligned}
$$

3. WINDING NUMBERS

We first introduce some special monogenic differential forms which are in a natural way associated to chains in $\mathbb{R}^{m}$.

Definition 3. (i) Let $\Sigma$ be an $(m-k)$-chain in $\mathbb{R}^{m}$. Then the (left) indicatrix of $\Sigma$ is the left monogenic $k$-form

$$
I_{l}(\Sigma)(\mathbf{x})=\int_{\mathbf{y} \in \Sigma} E(\mathbf{x}-\mathbf{y}) d V_{k}(\mathbf{x}, \mathbf{y}) .
$$

(ii) Let $\Sigma$ be an $(m-k-1)$-chain in $\mathbb{R}^{m}$. Then the (left) directed indicatrix of $\Sigma$ is the left monogenic $k$-form

$$
\left.J_{l}(\Sigma)(\mathbf{x})=\int_{\mathbf{y} \in \Sigma} E(\mathbf{x}-\mathbf{y}) d V_{k}(\mathbf{x}, \mathbf{y})\right\rfloor \partial_{\mathbf{y}}
$$

(iii) For a $k$-chain (resp. $(k-1)$-chain), the right indicatrix (resp. directed indicatrix) is given by

$$
I_{r}(\Sigma)(\mathbf{y})=\int_{\mathbf{x} \in \Sigma} E(\mathbf{x}-\mathbf{y}) d V_{k}(\mathbf{x}, \mathbf{y})
$$

(resp. $\left.\left.J_{r}(\Sigma)(\mathbf{y})=\int_{\mathbf{x} \in \Sigma} \partial_{\mathbf{x}}\right\rfloor d V_{k}(\mathbf{x}, \mathbf{y}) E(\mathbf{x}-\mathbf{y})\right)$.

Before studying the indicatrices of chains we prove the following technical

Lemma 2. Let $f$ be any distribution. Then

$$
d_{\mathbf{x}} \wedge\left(f(\mathbf{x}-\mathbf{y}) d V_{k}(\mathbf{x}, \mathbf{y})\right)=(-1)^{m}\left(f(\mathbf{x}-\mathbf{y}) d V_{k+1}(\mathbf{x}, \mathbf{y})\right) \wedge d_{\mathbf{y}}
$$


Proof. We have that

$$
\begin{aligned}
d_{\mathbf{x}} \wedge\left(f(\mathbf{x}-\mathbf{y}) d V_{k}(\mathbf{x}, \mathbf{y})\right) & =\sum_{|A|=k} \sum_{j=1}^{m} \operatorname{sgn} A d x_{j} \wedge d x_{A} d y_{M \backslash A} \partial_{x_{j}} f(\mathbf{x}-\mathbf{y}) \\
& =\sum_{\alpha_{1}<\cdots<\alpha_{k+1}} d x_{\alpha_{1}} \wedge \cdots \wedge d x_{\alpha_{k+1}} G_{\alpha_{1} \cdots \alpha_{k+1}},
\end{aligned}
$$

where for $A=\left\{\alpha_{1}, \ldots, \alpha_{k+1}\right\}, A_{j}=A \backslash\left\{\alpha_{j}\right\}$,

$$
G_{A}=-\sum_{j=1}^{k+1}(-1)^{j+1} \operatorname{sgn} A_{j} d y_{M \backslash A_{j}} \partial_{y_{\alpha_{j}}} f(\mathbf{x}-\mathbf{y}) .
$$

Now put $d y_{M \backslash A_{j}}=\kappa d y_{M \backslash A} \wedge d y_{\alpha_{j}} ;$ then

$$
\begin{aligned}
\operatorname{sgn} A & =\operatorname{sgn}\left\{\alpha_{1}, \ldots, \alpha_{k+1}, \beta_{1}, \ldots, \beta_{m-k-1}\right\} \\
& =(-1)^{k-j+1} \operatorname{sgn}\left\{A_{j}, \alpha_{j}, \beta_{1}, \ldots, \beta_{m-k-1}\right\} \\
& =(-1)^{k-j+1}(-1)^{m-k-1} \operatorname{sgn}\left\{A_{j}, M \backslash A, \alpha_{j}\right\}=(-1)^{m-j} \operatorname{sgn} A_{j} \kappa .
\end{aligned}
$$

Hence

$$
G_{A}=(-1)^{m} \sum_{j=1}^{k+1} \operatorname{sgn} A d y_{M \backslash A} \wedge d y_{\alpha_{j}} \partial_{y_{\alpha_{j}}} f(\mathbf{x}-\mathbf{y}) .
$$

In general, the indicatrices of chains are not closed, but one has the following Lemma 3. Let $\Sigma$ be an $(m-k)$-chain (resp. an $(m-k-1)$-chain) and let $\Sigma^{\prime}$ be a $k$-chain (resp. $(k-1)$-chain) in $\mathbb{R}^{m}$. Then we have the identities

$$
\begin{aligned}
& d_{\mathbf{x}} \wedge I_{l}(\Sigma)(\mathbf{x})=(-1)^{k+1} I_{l}(\partial \Sigma)(\mathbf{x}), \\
& d_{\mathbf{x}} \wedge J_{l}(\Sigma)(\mathbf{x})=(-1)^{k} J_{l}(\partial \Sigma)(\mathbf{x}), \\
& I_{r}\left(\Sigma^{\prime}\right)(\mathbf{y}) \wedge d_{\mathbf{y}}=(-1)^{m} I_{r}\left(\partial \Sigma^{\prime}\right)(y), \\
& J_{r}\left(\Sigma^{\prime}\right)(\mathbf{y}) \wedge d_{\mathbf{y}}=(-1)^{m+1} J_{r}\left(\partial \Sigma^{\prime}\right)(y) .
\end{aligned}
$$

Proof. We only show the first and the last identity. By Lemma 2 and Stokes' theorem we have that

$$
\begin{aligned}
d_{\mathbf{x}} \int_{\mathbf{y} \in \Sigma} E(\mathbf{x}-\mathbf{y}) d V_{k}(\mathbf{x}, \mathbf{y}) & =(-1)^{m} \int_{\mathbf{y} \in \Sigma} E(\mathbf{x}-\mathbf{y}) d V_{k+1}(\mathbf{x}, \mathbf{y}) \wedge d_{\mathbf{y}} \\
& =(-1)^{m}(-1)^{m-k-1} I_{l}(\partial \Sigma)(\mathbf{x}),
\end{aligned}
$$

while

$$
\begin{aligned}
J_{r}\left(\Sigma^{\prime}\right) & \left.\wedge d_{\mathbf{y}}=(-1)^{m} \int_{\Sigma^{\prime}} \partial_{\mathbf{x}}\right\rfloor\left(d_{\mathbf{x}} \wedge d V_{k-1}(\mathbf{x}, \mathbf{y}) E(\mathbf{x}-\mathbf{y})\right) \\
& \left.=(-1)^{m+1}\left(\int_{\Sigma^{\prime}} d_{\mathbf{x}} \wedge \partial_{\mathbf{x}}\right\rfloor d V_{k-1}(\mathbf{x}, \mathbf{y}) E(\mathbf{x}-\mathbf{y})+\int_{\Sigma^{\prime}} d V_{k-1}(\mathbf{x}, \mathbf{y}) \delta(\mathbf{x}-\mathbf{y})\right) \\
& =(-1)^{m+1} J_{r}\left(\partial \Sigma^{\prime}\right) .
\end{aligned}
$$


Hence, the indicatrices are closed monogenic forms if and only if the corresponding chains are cycles.

Now let $\Sigma$ be an $(m-k)$-cyle in $\mathbb{R}^{m}$ and $\Sigma^{\prime}$ a $(k-1)$-cycle in $\mathbb{R}^{m} \backslash \Sigma$, i.e., $\Sigma \cap \Sigma^{\prime}=\varnothing$. Then $\left.\partial_{\mathbf{x}}\right\rfloor I_{l}(\Sigma)(\mathbf{x})$ is closed in $\mathbb{R}^{m} \backslash \Sigma$ and so, by Stokes' theorem, the quantity

$$
\left.I\left(\Sigma^{\prime}, \Sigma\right)=\int_{\Sigma^{\prime}} \partial_{\mathbf{x}}\right\rfloor I_{l}(\Sigma)(\mathbf{x})
$$

is invariant under continuous deformations of $\Sigma^{\prime}$ inside $\mathbb{R}^{m} \backslash \Sigma$. On the other hand it is clear that also

$$
I\left(\Sigma^{\prime}, \Sigma\right)=\int_{\Sigma} J_{r}\left(\Sigma^{\prime}\right)(\mathbf{y})
$$

Hence, as $J_{r}\left(\Sigma^{\prime}\right)$ is closed in $\mathbb{R}^{m} \backslash \Sigma^{\prime}, I\left(\Sigma^{\prime}, \Sigma\right)$ is equally well invariant under continuous deformations of $\Sigma$ inside $\mathbb{R}^{m} \backslash \Sigma^{\prime}$. Now the winding number $c\left(\Sigma^{\prime}, \Sigma\right)$ which characterizes the "number of times" that $\Sigma^{\prime}$ cycles around $\Sigma$, has a similar invariance property (see, e.g., [6]). Hence we expect a relation between $I\left(\Sigma^{\prime}, \Sigma\right)$ and $c\left(\Sigma^{\prime}, \Sigma\right)$ which is expressed in

Theorem 3 (Winding number theorm). Let $\Sigma$ be an $(m-1)$-cycle and $\Sigma^{\prime}$ a $(k-1)$-cycle in $\mathbb{R}^{m}$ such that $\Sigma \cap \Sigma^{\prime}=\varnothing$. Then the winding number $c\left(\Sigma^{\prime}, \Sigma\right)$ is, up to a signature, given by the double integral

$$
\left.I\left(\Sigma^{\prime}, \Sigma\right)=\int_{\mathbf{x} \in \Sigma^{\prime}} \int_{\mathbf{y} \in \Sigma} \partial_{\mathbf{x}}\right\rfloor d V_{k}(\mathbf{x}, \mathbf{y}) E(\mathbf{x}-\mathbf{y}) .
$$

Proof. The proof is similar to the one we already gave for Theorem 2.5 in [11] (see also [6-8]). First let $\Sigma^{\prime}=\partial \Sigma^{\prime \prime}$; then by a transversality argument, $\Sigma^{\prime \prime}$ can be chosen such that $\Sigma$ and $\Sigma^{\prime \prime}$ intersect in finitely many points. Then we can deform $\Sigma^{\prime}$ inside $\Sigma^{\prime \prime}$ to a disjoint union of finitely many sphere-like cycles $C_{j}$. Next, $\Sigma$ may be deformed in $\mathbb{R}^{m} \backslash \bigcup_{j} C_{j}$ to a union of sphere-like cycles. So it suffices to prove the theorem in the case where $\Sigma$ and $\Sigma^{\prime}$ are spheres and we may even deform $\Sigma$ into a subspace. Now let $\Sigma=\left\{\mathbf{y}: y_{1}=\cdots=y_{k}=0\right\}$. Then

$$
\begin{aligned}
I_{l}(\Sigma)(\mathbf{x}) & =\int_{\Sigma} d V_{k}(\mathbf{x}, \mathbf{y}) E(\mathbf{x}-\mathbf{y}) \\
& =\int_{\mathbb{R}^{m-k}} d x_{1} \wedge \cdots \wedge d x_{k} \frac{1}{\omega_{m}} \frac{\mathbf{x}-\mathbf{y}}{|\mathbf{x}-\mathbf{y}|^{m}} d y_{k+1} \cdots d y_{m} \\
& =\frac{d x_{1} \wedge \cdots \wedge d x_{k}}{\omega_{k}} \frac{\mathbf{x}}{|\mathbf{x}|^{k}} .
\end{aligned}
$$

Next, let $\Sigma^{\prime}$ be the sphere $x_{1}^{2}+\cdots+x_{k}^{2}=1$ in $\mathbb{R}^{k}=\Sigma^{\perp}$, provided with the "outer unit normal" orientation. Then

$$
\left.I\left(\Sigma^{\prime}, \Sigma\right)=\frac{1}{\omega_{k}} \int_{S^{k-1}} \partial_{\mathbf{x}}\right\rfloor I_{l}(\Sigma)(\mathbf{x})=\frac{1}{\omega_{k}} \int_{S^{k-1}} d \sigma_{\mathbf{x}} \frac{\mathbf{x}}{|\mathbf{x}|^{k}}=-1 .
$$

Remark. The winding number is obtained as the integral of $\left.\partial_{\mathbf{x}}\right\rfloor I_{l}(\Sigma)$ over the cycle $\Sigma^{\prime}$, where $\left.\partial_{\mathbf{x}}\right\rfloor I_{l}(\Sigma)$ is a closed $(k-1)$-form in $\mathbb{R}^{m} \backslash \Sigma$. But we have 
seen that $I_{l}(\Sigma)$ is also a closed $k$-form in $\mathbb{R}^{m} \backslash \Sigma$, so one may be tempted to consider integrals of the form $\int_{\Sigma^{\prime}} I_{l}(\Sigma)(\mathbf{x}), \Sigma^{\prime}$ a $k$-cycle. However, after suitable deformations of both $\Sigma$ and $\Sigma^{\prime}, \Sigma^{\prime}$ becomes equivalent to a boundary in $\mathbb{R}^{m} \backslash \Sigma$ and so $\int_{\Sigma^{\prime}} I_{l}(\Sigma)(\mathbf{x})=0$. By de Rham's theorem, this means that $I_{l}(\Sigma)(\mathbf{x})$ is an exact form in $\mathbb{R}^{m} \backslash \Sigma$ while $\left.\partial_{\mathbf{x}}\right\rfloor I_{l}(\Sigma)$ clearly is not exact!

\section{Monogenic COHOMOLOGY}

In the previous section we constructed indicatrices of cycles, which were monogenic forms. Hence it must be possible to construct explicit bases for the de Rham cohomology spaces with values in $\mathbf{C}_{m}$, denoted by $H_{\text {deRham }}^{k}\left(\Omega, \mathbf{C}_{m}\right)$, $\Omega \subseteq \mathbb{R}^{m}$ open, consisting of indicatrices of cycles or $\partial_{\mathrm{x}}$-contractions of them. So one can, e.g., construct a "monogenic basis" for the de Rham cohomology spaces. In the following theorem we give an independent proof of this, just using the fact that the equation $\partial_{\mathbf{x}} f=g$ is solvable in the space $\mathscr{E}\left(\Omega ; \mathbf{C}_{m}\right)$ of $\mathrm{C}_{m}$-valued $C_{\infty}$-functions in $\Omega$.

Theorem 4 (First monogenic cohomology theorem). Let $F$ be a smooth closed $k$-form in $\Omega \subseteq \mathbb{R}^{m}$. Then there exists a smooth $(k-1)$-form $G$ in $\Omega$ such that $F-d_{\mathbf{x}} G$ is left monogenic.

Proof. Let $F$ be given and put $\left.H=\partial_{\mathbf{x}}\right\rfloor F$. Then there exists a $(k-1)$-form $G$ such that $\partial_{\mathbf{x}} G=H$. Indeed, put $H=\sum_{A} d x_{A} H_{A}$; then it suffices to take $G=\sum_{A} d x_{A} G_{A}$, where $G_{A} \in \mathscr{E}\left(\Omega ; \mathbf{C}_{m}\right)$ satisfies $\partial_{\mathbf{x}} G_{A}=H_{A}$ (see [2]). Now $f-d_{\mathbf{x}} G$ is left monogenic. Indeed, as $F$ is closed, $\left.\partial_{\mathbf{x}} F=d_{\mathbf{x}} \wedge \partial_{\mathbf{x}}\right\rfloor F$, while on the other hand,

$$
\left.\partial_{\mathbf{x}}\left(d_{\mathbf{x}} \wedge G\right)=d_{\mathbf{x}} \wedge \partial_{\mathbf{x}} G=d_{\mathbf{x}} \wedge\left(\partial_{\mathbf{x}}\right\rfloor F\right)
$$

Notice that it follows from the first basic identities that $\partial_{\mathbf{x}}\left(d_{\mathbf{x}} \wedge G\right)=d_{\mathbf{x}} \wedge \partial_{\mathbf{x}} G$.

The previous theorem is of course directly related to the fact that when $G$ is closed, then $\left.\partial_{\mathbf{x}} G=d_{\mathbf{x}} \wedge \partial_{\mathbf{x}}\right\rfloor G$ is exact. Now when $G$ is monogenic and closed; then $\left.\partial_{\mathbf{x}}\right\rfloor G$ is still closed. When $G$ is exact then $\left.\partial_{\mathbf{x}}\right\rfloor G$ is in general not exact as the counterexample $G=I_{l}(\Sigma)$ shows. This example also suggests that we might try to represent the de Rham cohomology spaces by forms such as $\left.\partial_{\mathbf{x}}\right\rfloor G$, $G$ monogenic and exact. This problem is solved in

Theorem 5 (Second monogenic cohomology theorem). Let $F$ be a closed smooth $k$-form in $\Omega$. Then for some smooth $(k-1)$-form $G, F-d_{\mathbf{x}} G$ is of the form $\left.\partial_{\mathbf{x}}\right\rfloor H, H$ being an exact monogenic $(k+1)$-form in $\Omega$.

Proof. Let $L$ be a smooth solution of $\partial_{\mathbf{x}} L=F$ or $\left.\left.\partial_{\mathbf{x}}\right\rfloor\left(d_{\mathbf{x}} \wedge L\right)=F-d_{\mathbf{x}} \wedge\left(\partial_{\mathbf{x}}\right\rfloor L\right)$. Then it suffices to put $\left.G=\partial_{\mathbf{x}}\right\rfloor L$ and $H=d_{\mathbf{x}} \wedge L$. We only have to check that $\partial_{\mathbf{x}} H=0$. This follows from $\partial_{\mathbf{x}} H=\partial_{\mathbf{x}}\left(d_{\mathbf{x}} \wedge L\right)=d_{\mathbf{x}} \wedge \partial_{\mathbf{x}} L=d_{\mathbf{x}} F=0$.

If we apply this to a form $\left.\partial_{\mathbf{x}}\right\rfloor G, G$ closed and monogenic, we find that for some exact and monogenic form $\left.H, \partial_{\mathbf{x}}\right\rfloor(G-H)$ is exact, although $G-H$ need not be exact. Hence as to exactness there is no link between $F$ and $\left.\partial_{\mathbf{x}}\right\rfloor F$. 
Both cohomology theorems allow us to correct closed forms to either closed monogenic forms or forms like $\left.\partial_{\mathbf{x}}\right\rfloor H, H$ exact monogenic. Hence the elements of the de Rham cohomology spaces may be represented by differential forms of both types.

Next, let us denote by $M_{l}^{k}(\Omega)$ (resp. $\left.M_{r}^{k}(\Omega)\right)$ the space of left (resp. right) monogenic $k$-forms in $\Omega$. Then we can consider the complex

$$
0 \rightarrow M_{l}^{0}(\Omega) \stackrel{d_{0}}{\longrightarrow} M_{l}^{1}(\Omega) \rightarrow \cdots \rightarrow M_{l}^{m}(\Omega) \stackrel{d_{m}}{\longrightarrow} 0
$$

and we can introduce the "monogenic cohomology" spaces

$$
H_{l}^{k}(\Omega)=\operatorname{ker} d_{k} / \operatorname{im} d_{k-1},
$$

where $d_{k}=d \mid M_{l}^{k}(\Omega)$. The spaces $H_{l}^{k}(\Omega)$ are not isomorphic to the de Rham cohomology spaces. Indeed, if this were so, then in view of the first monogenic cohomology theorem, every exact monogenic form $H$ should be of the form $d_{\mathbf{x}} \wedge G, G$ monogenic. This cannot be true because then $\left.\partial_{\mathbf{x}}\right\rfloor H=$ $\left.\left.\partial_{\mathbf{x}}\right\rfloor\left(d_{\mathbf{x}} \wedge G\right)=-d_{\mathbf{x}} \wedge \partial_{\mathbf{x}}\right\rfloor G$ would always be exact, which violates the second cohomology theorem. This leads to the introduction of three different kinds of monogenic differential forms in the following

Definition 4. The space of closed left (right) monogenic $k$-forms in $\Omega$ is denoted by $M C_{l}^{k}(\Omega)$ (resp. $M C_{r}^{k}(\Omega)$ ). The space of exact left (right) monogenic $k$-forms in $\Omega$ is denoted by $M e_{l}^{k}(\Omega)$ (resp. $M e_{r}^{k}(\Omega)$ ). A monogenic $k$-form of type $d_{\mathbf{x}} \wedge G, G$ monogenic, is called strongly exact. The spaces of such forms are denoted by $M E_{l}^{k}(\Omega)$ and $M E_{r}^{k}(\Omega)$.

Now the first cohomology theorems says that

$$
H_{\text {deRham }}^{k}\left(\Omega ; \mathbf{C}_{m}\right) \cong M C_{l}^{k}(\Omega) / M e_{l}^{k}(\Omega) .
$$

The second cohomology theorem says that the above space may be represented by $\left.\partial_{\mathbf{x}}\right\rfloor M e_{l}^{k+1}(\Omega)$. Moreover, when $H$ is strongly exact, then $\left.\partial_{\mathbf{x}}\right\rfloor H$ is still exact. So we may wonder whether

$$
\left.\left.H_{\text {deRham }}^{k}\left(\Omega ; \mathbf{C}_{m}\right) \cong \partial_{\mathbf{x}}\right\rfloor M e_{l}^{k+1}(\Omega) / \partial_{\mathbf{x}}\right\rfloor M E_{l}^{k+1}(\Omega) .
$$

This will turn out to be true. In fact we have the following charcterization of strong exactness.

Lemma 4. $H$ is strongly exact if and only if both $H$ and $\left.\partial_{\mathbf{x}}\right\rfloor H$ are exact.

Proof. We already mentioned that $\left.\partial_{\mathbf{x}}\right\rfloor H$ is exact as soon as $H$ is strongly exact. Conversely assume that both $H$ and $\left.\partial_{\mathbf{x}}\right\rfloor H$ are exact. Then $H$ is clearly monogenic and $\left.H=d \wedge G, \partial_{\mathbf{x}}\right\rfloor H=d \wedge F$ for some differential forms $G$ and $F$. But then

$$
\left.\left.\left.\partial_{\mathbf{x}} G=\partial_{\mathbf{x}}\right\rfloor H+d \wedge \partial_{\mathbf{x}}\right\rfloor G=d \wedge\left(F+\partial_{\mathbf{x}}\right\rfloor G\right)=d \wedge L .
$$

Now let $J$ be a solution of $\partial_{\mathbf{x}} J=L$; then $M=G-d \wedge J$ is monogenic and $H=d \wedge M$.

These ingredients all lead to the main theorem. 
Theorem 6 (Monogenic cohomology theorem). There exists a natural isomorphism

$$
\theta: H_{l}^{k}(\Omega) \rightarrow H_{\mathrm{deRham}}^{k}\left(\Omega ; \mathbf{C}_{m}\right) \oplus H_{\mathrm{deRham}}^{k-1}\left(\Omega ; \mathbf{C}_{m}\right) .
$$

Proof. Let $F \in H_{l}^{k}(\Omega)$. Then we define $\left.\theta(F)=\left(F, \partial_{\mathbf{x}}\right\rfloor F\right)$, where it is understood that $\left.\left(F, \partial_{\mathbf{x}}\right\rfloor F\right)$ is an element of $H_{\text {deRham }}^{k}\left(\Omega ; \mathbf{C}_{m}\right) \oplus H_{\text {deRham }}^{k-1}\left(\Omega ; \mathbf{C}_{m}\right)$. This map is clearly well defined because when $F$ is monogenic and closed, then both $F$ and $\left.\partial_{\mathbf{x}}\right\rfloor F$ are closed, and when $F$ is strongly exact, then both $F$ and $\left.\partial_{\mathbf{x}}\right\rfloor F$ are exact.

The map $\theta$ is also injective because when $F$ and $\left.\partial_{\mathbf{x}}\right\rfloor F$ are exact, then $F$ is strongly exact. It remains to be shown that $\theta$ is onto. Let $G$ be a closed $k$-form and $H$ a closed $(k-1)$-form. By the first monogenic cohomology theorem, there exists a monogenic $k$-form $M$ such that $M=G$ in $\left.H_{\text {deRham }}^{k}(\Omega) . \partial_{\mathbf{x}}\right\rfloor M$ is also closed and so, by the second monogenic cohomology theorem, there exists an exact monogenic form $L$ such that in $\left.\left.H_{\text {deRham }}^{k-1}\left(\Omega ; \mathbf{C}_{m}\right), \partial_{\mathbf{x}}\right\rfloor L=H-\partial_{\mathbf{x}}\right\rfloor M$. Now put $F=M+L$; then $\theta(F)=(M+L, H)=(M, H)=(G, H)$.

\section{MONOGENIC CURRENTS AND FLOWS}

We first introduce some new definitions and notations.

Definition 5. (i) An element $T$ of the dual of the left $\mathbf{C}_{m}$-module $M_{r}^{m-k}(\Omega)$ is called a (left) monogenic $k$-current in $\Omega$. The action of $T \in M_{r}^{m-k}(\Omega)^{\prime}$ on $F \in M_{r}^{m-k}(\Omega)$ is denoted by $\langle F, T\rangle$.

(ii) A monogenic current $T$ is called closed if it vanishes on $M E_{r}^{m-k}(\Omega)$, i.e., $\langle F, T\rangle=0$ for every strongly exact form $F$. A closed monogenic current is called exact (resp. strongly exact) if it vanishes on $M e_{r}^{m-k}(\Omega)$ (resp. $\left.M C_{r}^{m-k}(\Omega)\right)$.

Notice that we preferred the somewhat nonstandard notation $\langle F, T\rangle$ instead of $\langle T, F\rangle$ for the action of $T$ on $F$. We did this because $M_{r}^{m-k}(\Omega)$ is a left $\mathrm{C}_{m}$-module and so $M_{r}^{m-k}(\Omega)^{\prime}$ is a right one and it would be awkward to write, e.g., $\langle T \lambda, a F\rangle=a\langle T, F\rangle \lambda$. Moreover, when $F=f$ is a 0 -form, and $\mu$ a $\mathbf{C}_{m}$-valued measure with compact support, then $\int f d \mu$ defines a current $T_{\mu}$ while $\int d \mu f$ does not. So it is better to use the notion $\left\langle f, T_{\mu}\right\rangle$ instead of $\left\langle T_{\mu}, f\right\rangle$ for this.

In a way similar to Definition 5, one can introduce right monogenic currents. The action of such a current $T$ on a left monogenic differential form $F$ will now be denoted by $\langle T, F\rangle$.

Let $A(\Omega)$ be one of the above spaces of left or right monogenic differential forms; then we will use the notation

$$
A(K)=\operatorname{limind}_{K \subset \Omega} A(\Omega), \quad K \subseteq \mathbb{R}^{m} \text { compact },
$$

and the dual of $A(K)$ will be denoted by $A^{\prime}(K)$. 
It is easy to see that $T \in M_{r}^{m-k}(\Omega)^{\prime}$ belongs to some $M_{r}^{m-k}\left(K^{\prime}\right)$ and so one could think of monogenic currents as compactly supported objects. However, there is no real notion of support for currents. The only thing one can do is to define carriers.

Definition 6. Let $T \in M_{r}^{m-k}(\Omega)^{\prime}$ and $K \subseteq \Omega$ compact. Then $K$ is called a carrier of $T$ if $M_{r}^{m-k}(\Omega)$ is dense in $M_{r}^{m-k}(K)$, if $T \in M_{r}^{m-k}(K)^{\prime}$, and if $K$ is minimal with respect to these properties.

As the case $k=m$ shows, $T$ may well have several carriers. By Runge's theorem (see [2]), $M_{r}^{m-k}(\Omega)$ is dense in $M_{r}^{m-k}(K)$ if and only if every component of $\Omega \backslash K$ contains points of $\partial \Omega$. The cases $k=0$ and $k=m$ correspond to the theory of so-called monogenic functionals, first introduced in [3], for which we already introduced several integral transforms (see also [10]). In particular one can show that compactly supported hyperfunctions or Schwartz-distributions are special examples of monogenic functionals (see $[2,10])$. In the present theory of currents there are several other natural examples, which are not incorporated in the theory of monogenic functionals of [3]. The following ones are of basic importance.

Examples. (1) Let $\Sigma \subseteq \Omega$ be an $(m-k)$-chain. Then the Dirac current $\delta_{\Sigma}$ on $\Sigma$ is given by

$$
\left\langle F, \delta_{\Sigma}\right\rangle=\int_{\Sigma} F(\mathbf{x}) .
$$

For $k=m, \Sigma$ is a union of points in which $F$ is to be evaluated. Unless $k=0, \delta_{\Sigma}$ is carried by $\Sigma$ and for $k>1$ the carrier is unique. When $\Sigma$ is a cycle in $\Omega$, then $\delta_{\Sigma}$ is exact. When $\Sigma$ is a boundary in $\Omega$, then $\delta_{\Sigma}$ is strongly exact.

(2) Let $\Sigma \subseteq \Omega$ be an $(m-k-1)$-chain. Then the directed Dirac current $\boldsymbol{\delta}_{\Sigma}$ is given by

$$
\left.\left\langle F, \boldsymbol{\delta}_{\Sigma}\right\rangle=\int_{\Sigma} F(\mathbf{x})\right\rfloor \partial_{\mathbf{x}} .
$$

Unless $k=0, \boldsymbol{\delta}_{\Sigma}$ is carried by $\Sigma$. This is still true for $k=0$, provided that $\Sigma$ "contains" no boundaries (for $k=0, F(\mathbf{x})\rfloor \partial_{\mathbf{x}}$ is closed). When $k=0$ and $\Sigma-\Sigma^{\prime}$ is a boundary, then $\delta_{\Sigma}=\delta_{\Sigma^{\prime}}$. So the carrier is not unique. When $\Sigma$ is a cycle, then $\boldsymbol{\delta}_{\Sigma}$ is closed but need not be exact ( $F$ exact does not imply $F(\mathbf{x})\rfloor \partial_{\mathbf{x}}$ exact).

To make the link between monogenic currents and differential forms we introduce the "indicatrix" or "field" of a current in

Definition 7. Let $T \in M_{r}^{m-k}(K)^{\prime}$. Then the field of $T$ is the left monogenic $k$-form in $\mathbb{R}^{m} \backslash K$.

$$
\widehat{T}(\mathbf{x})=(-1)^{(k-1)(m-k)}\left\langle d V_{k}(\mathbf{x}, \mathbf{y}) E(\mathbf{x}-\mathbf{y}), T_{\mathbf{y}}\right\rangle .
$$


Notice that $\widehat{T}(\infty)=0$. The following theorem generalizes the duality theorem in [3]. $\quad M_{0, l}^{k}\left(\mathbb{R}^{m} \backslash K\right)$ denotes the space of left monogenic $k$-forms in $\mathbb{R}^{m} \backslash K$, vanishing at infinity. We have

Theorem 7. The map $T \rightarrow \widehat{T}$ defines an isomorphism between $M_{r}^{m-r}(K)^{\prime}$ and $M_{0, l}^{k}\left(\mathbb{R}^{m} \backslash K\right)$.

Proof. Let $T \in M_{r}^{m-k}(K)^{\prime}$ and $F \in M_{r}^{m-k}(\Omega), K \subseteq \Omega$, and take a closed set $C \subseteq \Omega$ with $C_{1}$-boundary such that $K \subset \stackrel{\circ}{C}$. Then by Theorem 2,

$$
\begin{array}{rl}
\int_{\partial C} & F(\mathbf{x}) \diamond \widehat{T}(\mathbf{x})=(-1)^{(k-1)(m-k)} \int_{\partial C} F(\mathbf{x}) \diamond\left\langle d V_{k}(\mathbf{x}, \mathbf{y}) E(\mathbf{x}-\mathbf{y}), T_{\mathbf{y}}\right\rangle \\
= & (-1)^{(k-1)(m-k)}\left\langle\int_{\partial C} F(\mathbf{x}) \diamond d V_{k}(\mathbf{x}, \mathbf{y}) E(\mathbf{x}-\mathbf{y}), T_{\mathbf{y}}\right\rangle \\
& =\left\langle F(\mathbf{y}), T_{\mathbf{y}}\right\rangle .
\end{array}
$$

Moreover, in general, for $G \in M_{0, l}^{k}\left(\mathbb{R}^{m} \backslash K\right)$ we may introduce

$$
R s G \in M_{r}^{(m-k)}(K)
$$

by

$$
\langle F, R s G\rangle=\int_{\partial C} F(\mathbf{x}) \diamond G(\mathbf{x}) .
$$

Clearly $G=\widehat{R S G}$.

The proof of the duality theorem leads to the following

Definition 8. Let $G \in M_{l}^{k}\left(\mathbb{R}^{m} \backslash K\right)$. Then the "Grand Residue" $R s G$ of $G$ is the left monogenic $k$-current determined by

$$
\langle F, R s G\rangle=\int_{\partial C} F(\mathbf{x}) \diamond G(\mathbf{x}),
$$

where $F$ and $C$ are as in the previous proof.

The above introduced notion of residue is not quite the same as the classical notion of residue of a holomorphic function although it is closely related to it. Indeed, let $f(z)$ and $g(z)$ be holomorphic and put $G(\mathbf{x})=d x d y g(z)$, $F(\mathbf{x})=f(z) ;$ then

$$
\left.F \diamond G=f(z)\left(\partial_{x}+i \partial_{y}\right)\right\rfloor d x \wedge d y g(z)=\frac{1}{i} f(z) g(z) d z,
$$

where $f(z)=\sum_{j=0}^{\infty} a_{j} z^{j}$ and $g(z)=g_{1}(z)+\sum_{j=0}^{\infty} c_{j} z^{-j-1}$. Then we have that $\langle F, R s G\rangle=2 \pi \sum_{j=0}^{\infty} a_{j} c_{j}$ so that in fact

$$
\text { Rs } G=2 \pi \sum_{j=0}^{\infty} \frac{(-1)^{j} c_{j}}{j !} \delta^{(j)} \text {. }
$$

Hence $R s G$ contains not only the residue of $g$, but all Laurent coefficients. In the following theorem we characterize closed exact and strongly exact currents. 
Theorem 8. A monogenic current $T$ is closed, exact or strongly exact, if and only if its field $\widehat{T}$ has the corresponding property.

Proof. Let $T$ be closed. Then by Lemma 2, for some $\sigma= \pm 1$,

$$
d_{\mathbf{x}} \wedge \widehat{T}(\mathbf{x})=\sigma\left\langle d V_{k+1}(\mathbf{x}, \mathbf{y}) E(\mathbf{x}-\mathbf{y}) \wedge d_{\mathbf{y}}, T_{\mathbf{y}}\right\rangle=0,
$$

because the form on which $T$ acts in the above formula is clearly strongly exact. Conversely, let $G \in M_{0, l}^{k}\left(\mathbb{R}^{m} \backslash K\right)$ be closed and $F \in M_{r}^{m-k}(K)$ be strongly exact with $F=d_{\mathbf{x}} \wedge H$. Then $F \diamond G=\left(d_{\mathbf{x}} H\right) \diamond G=d_{\mathbf{x}}(H \diamond G)$, so that

$$
\langle F, R s G\rangle=\int_{\partial C} F \diamond G=0 .
$$

Next, let $T$ be strongly exact and let $C \subseteq \mathbb{R}^{m} \backslash K$ be any $k$-cycle. Then for $\sigma= \pm 1$

$$
\begin{aligned}
\int_{C} \widehat{T}(\mathbf{x}) & =\sigma\left\langle\int_{x \in C} d V_{k}(\mathbf{x}, \mathbf{y}) E(\mathbf{x}-\mathbf{y}), T_{\mathbf{y}}\right\rangle \\
& =\sigma\left\langle I_{r}(C)(\mathbf{y}), T_{\mathbf{y}}\right\rangle=0,
\end{aligned}
$$

because $I_{r}(C)$ is closed and even exact. Similarly, for any $(k-1)$-cycle $C \subseteq$ $\mathbb{R}^{m} \backslash K$,

$$
\left.\int_{C} \partial_{\mathbf{x}}\right\rfloor \widehat{T}(\mathbf{x})=\sigma\left\langle J_{r}(C)(\mathbf{y}), T_{\mathbf{y}}\right\rangle=0,
$$

because $J_{r}(C)$ is also closed. Hence, by de Rham's theorem, $\widehat{T}(\mathbf{x})$ and $\left.\partial_{\mathbf{x}}\right\rfloor \widehat{T}$ are both exact, which by Lemma 4 means that $\widehat{T}(\mathbf{x})$ is strongly exact. Conversely, let $G \in M_{0, l}^{k}\left(\mathbb{R}^{m} \backslash K\right)$ be strongly exact and put, e.g., $G=d_{\mathbf{x}} \wedge H, H$ monogenic. Then for $F \in M C_{r}^{m-k}(K)$,

$$
\langle F, R s G\rangle=\int_{C} F \diamond\left(d_{\mathbf{x}} \wedge H\right)=\int_{C} d_{\mathbf{x}}(F \diamond H)=0 .
$$

Finally, let $T$ be exact and $C \subseteq \mathbb{R}^{m} \backslash K$ a $k$-cycle; then

$$
\int_{C} \widehat{T}(\mathbf{x})=\sigma\left\langle I_{r}(C)(\mathbf{y}), T_{\mathbf{y}}\right\rangle=0,
$$

since $I_{r}(C)$ is exact. Hence by de Rham's theorem, $\widehat{T}$ is exact. Conversely, let $G \in M_{0, l}^{k}\left(\mathbb{R}^{m} \backslash K\right)$ and $F \in M_{r}^{m-k}(K)$ both be exact and put $G=d_{\mathbf{x}} \wedge L$ and $F=d_{\mathbf{x}} \wedge M$; then $\partial_{\mathbf{x}} L$ and $M \partial_{\mathbf{x}}$ are closed. Hence we have

$$
\begin{aligned}
F \diamond G & =\left(d_{\mathbf{x}} \wedge M\right) \diamond G \\
& =d_{\mathbf{x}}(M \diamond G)+(-1)^{k}\left[M \diamond d_{\mathbf{x}} G+M \partial_{\mathbf{x}} \wedge G+M \wedge \partial_{\mathbf{x}} G\right] \\
& =d_{\mathbf{x}}(M \diamond G)+(-1)^{k} M \partial_{\mathbf{x}} \wedge\left(d_{\mathbf{x}} L\right)=d_{\mathbf{x}}\left(M \diamond G-M \partial_{\mathbf{x}} \wedge L\right),
\end{aligned}
$$

so that clearly $\langle F, R s G\rangle=\int_{C} F \diamond G=0$.

The previous theorem leads to the characterization of the duals of $M C_{r}^{m-k}(K), M e_{r}^{m-k}(K)$, and $M E_{r}^{m-k}(K)$. To that end, let $M C_{0, l}^{k}\left(\mathbb{R}^{m} \backslash K\right)$, $M e_{0, l}^{k}\left(\mathbb{R}^{m} \backslash K\right)$, and $M E_{0, l}^{k}\left(\mathbb{R}^{m} \backslash K\right)$ be the subspaces of $M_{0, l}^{k}\left(\mathbb{R}^{m} \backslash K\right)$ consisting of closed, exact, and strongly exact forms. Then we have the following theorem. 
Theorem 9. The map $T \rightarrow \widehat{T}$ leads to the isomorphisms

$$
\begin{gathered}
M C_{r}^{m-k}(K)^{\prime} \cong M_{0, l}^{k}\left(\mathbb{R}^{m} \backslash K\right) / M E_{0, l}^{k}\left(\mathbb{R}^{m} \backslash K\right), \\
M e_{r}^{m-k}(K)^{\prime} \cong M_{0, l}^{k}\left(\mathbb{R}^{m} \backslash K\right) / M e_{0, l}^{k}\left(\mathbb{R}^{m} \backslash K\right), \\
M E_{r}^{m-k}(K)^{\prime} \cong M_{0, l}^{k}\left(\mathbb{R}^{m} \backslash K\right) / M C_{0, l}^{k}\left(\mathbb{R}^{m} \backslash K\right) .
\end{gathered}
$$

Proof. Let, e.g., $T \in M C_{r}^{m-k}(K)^{\prime}$. Then as $M C_{r}^{m-k}(K)$ is a closed subspace of $M_{r}^{m-k}(K)$, by Hahn-Banach's theorem there exists an extension $S \in M_{r}^{m-k}(K)^{\prime}$ of $T$. We now put $\widehat{T}=\widehat{S}$. Then $\widehat{T}$ is determined up to a function $g \in$ $M_{0, l}^{k}\left(\mathbb{R}^{m} \backslash K\right)$ for which $R s g$ vanishes on $M C_{r}^{m-k}(K)$ or, in other words, $R s g$ is strongly exact. By Theorem 8 this is equivalent to $g \in M E_{0, l}^{k}\left(\mathbb{R}^{m} \backslash K\right)$. The other isomorphisms are similar.

Definition 9. An element $T \in M C_{r}^{m-k}(\Omega)^{\prime}$ is called a left monogenic $k$-flow in $\Omega$. $T$ is called closed or exact if $T$ vanishes respectively on the subspaces $M E_{r}^{m-k}(\Omega)$ and $M e_{r}^{m-k}(\Omega)$.

Notice that for $k=0$, every monogenic $k$-current is automatically a $k$-flow. The notion of carrier may as well be defined for $k$-flows and it is even more flexible than for $k$-currents.

Examples. (1) Let $\Sigma \subseteq \Omega$ be an $(m-k)$-chain. Then

$$
\widehat{\delta}_{\Sigma}(\mathbf{x})=(-1)^{(k-1)(m-k)} I_{l}(\Sigma)(\mathbf{x}) .
$$

When $\Sigma^{\prime}$ is another $(m-k)$-chain such that $\Sigma-\Sigma^{\prime}$ is a boundary in $\Omega$, then $\delta_{\Sigma}-\delta_{\Sigma}^{\prime}$ is strongly exact and therefore $\delta_{\Sigma}=\delta_{\Sigma^{\prime}}$ as $k$-flows. In most "normal situations", $\delta_{\Sigma}$ is carried by $\Sigma$ and hence also by $\Sigma^{\prime}$.

(2) Let $\Sigma \subseteq \Omega$ be an $(m-k-1)$-chain. Then clearly

$$
\widehat{\boldsymbol{\delta}}_{\Sigma}(\mathbf{x})=(-1)^{(k-1)(m-k)} J_{l}(\Sigma)(\mathbf{x}) .
$$

Again when $\Sigma-\Sigma^{\prime}$ is a boundary in $\Omega$, then $\boldsymbol{\delta}_{\Sigma}=\boldsymbol{\delta}_{\Sigma^{\prime}}$, as $k$-flows.

Notice that by Theorem 9, a closed $k$-flow $T$ has a field

$$
\widehat{T} \in M C_{0, l}^{k}\left(\mathbb{R}^{m} \backslash K\right) / M E_{0, l}^{k}\left(\mathbb{R}^{m} \backslash K\right),
$$

which is naturally isomorphic to the monogenic cohomology space $H_{l}^{k}(\Omega), \Omega=$ $\mathbb{R}^{m} \backslash K$. By the monogenic cohomology theorem, $H_{l}^{k}(\Omega)$ is itself isomorphic to the direct sum $H_{\text {deRham }}^{k}(\Omega) \oplus H_{\text {deRham }}^{k-1}(\Omega)$. Hence each element belonging to this direct sum may be represented by a uniquely determined closed monogenic flow.

\section{COMPARISON WITH OTHER THEORIES}

In this section we will show how our previous theory of monogenic differential forms, established in [11] and further elaborated in [12], may be considered as 
a special case of the present theory of monogenic differential forms. It will depend on the choice of a special direction in $\mathbb{R}^{m}$ (in fact in $\mathbb{R}^{m+1}$ ).

In our paper [11], we started with the operator

$$
D_{x}=\sum_{j=0}^{m} \varepsilon_{j} \partial_{x_{j}}=\partial_{x_{0}}+\sum_{j=1}^{m} \varepsilon_{j} \partial_{x_{j}}, \quad \text { where } \varepsilon_{j} \varepsilon_{k}+\varepsilon_{k} \varepsilon_{j}=-2 \delta_{i j} .
$$

Our ideas of how to construct monogenic differential forms were inspired by the holomorphic differential form $d z f(z), d z=d x+i d y$. Starting from the basic differential forms

$$
d z_{j}=d x_{j}-\varepsilon_{j} d x_{0}
$$

we constructed, for any $A=\left\{\alpha_{1}, \ldots, \alpha_{k}\right\} \subseteq M$ with $\alpha_{1}<\cdots<\alpha_{k}$, the differential forms $\sum_{A} d z_{A} G_{A}$, where $d z_{A}=d z_{\alpha_{1}} \wedge \cdots \wedge d z_{\alpha_{k}}$. Such forms were called left $k$-forms and every $k$-form may be written in a unique way as

$$
F=G^{(k)}+d x_{0} \wedge G^{(k-1)}
$$

$G^{(k)}$, resp. $G^{(k-1)}$, being left $k$-forms, resp. left $(k-1)$-forms. In particular we have

$$
\begin{gathered}
d=\partial+d x_{0} D_{x}, \quad \partial=\sum_{j=1}^{m} d z_{j} \partial_{x_{j}}, \\
d \wedge F=\partial \wedge G^{(k)}+d x_{0} \wedge\left(D_{x} G^{(k)}-\partial \wedge G^{(k-1)}\right) .
\end{gathered}
$$

A left $k$-form was called left monogenic if $d x_{0} \wedge D_{x} G^{(k)}=0$. Hence left monogenicity was in fact equivalent to $d \wedge G^{(k)}=\partial \wedge G^{(k)}$. In particular, for $k=m, \partial \wedge G^{(m)}=0$, so that a left $m$-form is monogenic if and only if it is closed. Notice that

$$
d z_{1} \wedge \cdots \wedge d z_{m}=\sum_{j=0}^{m}(-1)^{j} \varepsilon_{j} d \widehat{x}_{j}=d \sigma
$$

so that for $k=m$ we reobtained the standard monogenic $m$-forms $d \sigma f(x)$. In our paper we have also shown that for any open set $\Omega \subseteq \mathbb{R}^{m+1}$,

$$
H_{\text {deRham }}^{k}(\Omega)=\operatorname{ker} \partial\left|M^{k}(\Omega) / \operatorname{im} \partial\right| M^{k-1}(\Omega),
$$

so that in this theory the monogenic cohomology spaces exactly coincide with the de Rham cohomology spaces. We also obtained explicit formulae for winding numbers.

Now let us point out how the old theory fits into the new one. To that end, let $\partial_{\mathbf{x}}=\sum_{j=0}^{m} e_{j} \partial_{x_{j}}$ be the $(m+1)$-dimensional Dirac operator, where $e_{i} e_{j}+e_{j} e_{i}=-2 \delta_{i j}$ for all $i, j=0, \ldots, m$. When we put $\varepsilon_{j}=-e_{0} e_{j}$, then $\left\{\varepsilon_{1}, \ldots, \varepsilon_{m}\right\}$ generates $\mathbf{C}_{m}$ and we have that $D_{x}=-e_{0} \partial_{\mathbf{x}}$. Now it is easy to see that for any $A=\left\{\alpha_{1}, \ldots, \alpha_{k}\right\} \subseteq M$,

$$
\left.d z_{A}=d z_{\alpha_{1}} \wedge \cdots \wedge d z_{\alpha_{k}}=\sum_{j=0}^{k}(-1)^{j} \varepsilon_{j} d x_{\bar{A} \backslash\left\{\alpha_{j}\right\}}=D_{x}\right\rfloor d x_{\bar{A}},
$$


where $\bar{A}=\{0\} \cup A, \varepsilon_{0}=1, \alpha_{0}=0$. Hence, as $d x_{\bar{A}}=d x_{0} \wedge d x_{A}$, left monogenic $k$-forms in the old sense are of the form

$$
\left.G^{(k)}=D_{\mathbf{x}}\right\rfloor H, \quad H=\sum_{A} d x_{\bar{A}} G_{A}=d x_{0} \wedge \sum_{A} d x_{A} G_{A},
$$

where $H$ is a special type of $(k+1)$-form, left monogenic in the new sense. Looking back to the proof of the monogenic cohomology theorem, in view of $\left.G^{(k)}=-e_{0} \partial_{\mathbf{x}}\right\rfloor H$, it should be no surpise that the monogenic cohomology spaces in the old sense are certainly not bigger than the classical de Rham spaces. Of course the above calculations clearly show that the old theory depended on the choice of a special direction, namely the $e_{0}$-direction. But perhaps such a choice is necessary to get rid of the splitting of monogenic cohomology spaces, since, only because of its special form, $H$ is completely determined by $G^{(k)}$.

Next, let us consider the partially worked out constructions in the paper [4] by $\mathrm{K}$. Habetha. The author started from the Hodge *-operator

$$
\left(d x_{\alpha_{0}} \wedge \cdots \wedge d x_{\alpha_{k}}\right)^{*}=\operatorname{sgn}\left(\alpha_{0}, \ldots, \alpha_{k}, \beta_{k+1}, \ldots, \beta_{m}\right) d x_{\beta+1} \wedge \cdots \wedge d x_{\beta_{m}},
$$

so that in fact

$$
d z^{*}=\left(\sum_{j=0}^{m} \varepsilon_{j} d x_{j}\right)^{*}=d \sigma .
$$

Hence he was able to compute winding numbers for $m$-cycles in $\mathbb{R}^{m+1}$, using $d z^{*} E(x-y), E$ the Cauchy kernel. Although he did not develop similar winding number formulae for $k$-cycles, he did introduce the special differential forms

$$
\omega_{\alpha_{0} \cdots \alpha_{p}}=\left(d x_{\alpha_{0}} \wedge \cdots \wedge d x_{\alpha_{p}} \wedge d z\right)^{*}
$$

for which the following identity holds for monogenic functions:

$$
d\left(\omega_{\alpha_{0}, \ldots, \alpha_{p}} f\right)=\sum_{j=0}^{p}(-1)^{p-j+1} \omega_{\alpha_{0} \cdots \widehat{\alpha}_{j} \cdots \alpha_{p}} \partial_{x_{\alpha_{j}}} f .
$$

Now let us work in $\mathbb{R}^{m}$ instead of $\mathbb{R}^{m+1}$; consider $A=\left\{\alpha_{1}, \ldots, \alpha_{k}\right\} \subseteq M$ and $B=\left\{\beta_{1}, \ldots, \beta_{m-k}\right\}$ with $\alpha_{1}<\cdots<\alpha_{k}, \beta_{1}<\cdots<\beta_{m-k}$, and $M=B \cup A$. Then applying Habetha's definition,

$$
\begin{aligned}
\omega_{B} & =\left(d x_{\beta_{1}} \wedge \cdots \wedge d x_{\beta_{m-k}} \wedge d z\right)^{*} \\
& =\left(d x_{\beta_{1}} \wedge \cdots \wedge d x_{\beta_{m-k}} \sum_{j=1}^{k} e_{\alpha_{j}} d x_{\alpha_{j}}\right)^{*} \\
& =\sum_{j=1}^{k} e_{\alpha_{j}} \operatorname{sgn}\left(\beta_{1}, \ldots, \beta_{m-k}, \alpha_{j}, \alpha_{1}, \ldots, \hat{\alpha}_{j}, \ldots, \alpha_{k}\right) d x_{A \backslash\left\{\alpha_{j}\right\}} \\
& \left.=\operatorname{sgn} B \sum_{j=1}^{k} e_{\alpha_{j}}(-1)^{j+1} d x_{A \backslash\left\{\alpha_{j}\right\}}=\operatorname{sgn} B \cdot \partial_{\mathbf{x}}\right\rfloor d x_{A} .
\end{aligned}
$$


Furthermore when $f$ is left monogenic, then by the first basic identity,

$$
\begin{aligned}
d\left(\omega_{B} f\right) & \left.\left.=\operatorname{sgn} B d\left(\partial_{\mathbf{x}}\right\rfloor d x_{A} f\right)=-\operatorname{sgn} B \partial_{\mathbf{x}}\right\rfloor \sum_{j=1}^{m-k} d x_{\beta_{j}} \wedge d x_{A} \partial_{x_{\beta_{j}}} f \\
& =\sum_{j=1}^{m-k}(-1)^{p-j+1} \omega_{B \backslash\left\{\beta_{j}\right\}} \partial_{x_{\beta_{j}}} f .
\end{aligned}
$$

Hence our so-called first identity applied for monogenic differential forms, i.e., $\left.\left.d \wedge \partial_{\mathbf{x}}\right\rfloor F=-\partial_{\mathbf{x}}\right\rfloor(d F)$, is essentially equivalent to Habetha's lemma. So, at least part of the ideas leading to monogenic differential forms were already hidden in Habetha's paper [4]. It is interesting to notice that for our cohomological results, we only made use of the first basic identity, which defines $\partial_{\mathbf{x}} F$ as the Lie derivative $\left.\left.d\left(\partial_{\mathbf{x}}\right\rfloor F\right)+\partial_{\mathbf{x}}\right\rfloor(d F)$, together with the solvability of $\partial_{\mathbf{x}} F=G$ for smooth differential forms $F, G$ in open subsets of $\mathbb{R}^{m}$. Hence this part may be generalized to the case where $\partial_{\mathbf{x}}$ is a general $\mathbf{C}_{m}$-valued vector field for which $\partial_{\mathbf{x}} F=G$ is solvable. The second identity in Theorem 1 plays a central role in duality theory, leading to generalized residue calculus. In order to be able to compute winding numbers, one needs a "good" Cauchy kernel with point singularity, i.e., $\partial_{\mathbf{x}}$ should be elliptic. However, it might be interesting to try to develop winding number theory for nonelliptic operators $\partial_{\mathbf{x}}$ such as in the case of the complexified Dirac operator $\partial_{z}=\sum_{j=1}^{m} e_{j} \partial_{z_{j}}$, where one is dealing with holomorphic differential forms $F(\mathbf{z})$.

\section{REFERENCES}

1. J. W. Alexander, On the chains of a complex and their duals, Proc. Nat. Acad. Sci. U.S.A. 21 (1935), 509-511.

2. F. Brackx, R. Delanghe, and F. Sommen, Clifford anaylsis, Research Notes in Math., no. 76, Pitman, London, 1982.

3. R. Delanghe and F. Brackx, Duality in hypercomplex function theory, J. Funct. Anal. 37 (1978), 164-181.

4. K. Habetha, Eine Definition des Kroneckerindexes im $\mathbb{R}^{m+1}$ mit Hilfe der Cliffordanalysis, Z. Anal. Anwendungen 5 (1986), 133-137.

5. D. Hestenes and G. Sobczyk, Clifford algebra to geometric calculus, Reidel, Dordrecht, 1984.

6. M. W. Hirsch, Differential topology, Graduate Texts in Math., vol. 33, Springer, New York, 1976.

7. W. Hodge, The theory and applications of harmonic integrals, Cambridge Univ. Press, 1959.

8. L. S. Pontryagin, Foundations of combinatorial topology, Graylock Press, Rochester, 1952.

9. G. de Rham, Variétés différentiables. Formes, courants, formes harmoniques, Hermann, Paris, 1955.

10. F. Sommen, An extension of the Radon transform to Clifford analysis, Complex Variables Theory Appl. 8 (1987), 249-266.

11. 87-109.

12. F. Sommen and V. Souček, Hypercomplex differential forms applied to the de Rham and the Dolbeault complex, Sem. Geom. 1984, Univ. Bologna, 1985, pp. 177-192. 
13. V. Souček, Quaternion valued differential forms in $\mathbb{R}^{4}$, Suppl. Rend. Circ. Mat. Palermo (2) 33 (1984), 293-300.

14. C. von Westenholz, Differential forms in mathematical physics, Stud. Math. Appl., vol. 3 North-Holland, Amsterdam, 1978.

Seminar of Algebra and Functional Analysis, State University of Ghent, Galglaan 2, 9000 GENT, BELGIUM 